\title{
Engineering
}

\section{Bicuspid aortic valve aortopathies: An hemodynamics characterization in dilated aortas}

\section{Diana Oliveira, Sílvia Aguiar Rosa, Jorge Tiago, Rui Cruz Ferreira, Ana Figueiredo Agapito \& Adélia Sequeira}

To cite this article: Diana Oliveira, Sílvia Aguiar Rosa, Jorge Tiago, Rui Cruz Ferreira, Ana Figueiredo Agapito \& Adélia Sequeira (2019): Bicuspid aortic valve aortopathies: An hemodynamics characterization in dilated aortas, Computer Methods in Biomechanics and Biomedical Engineering, DOI: $10.1080 / 10255842.2019 .1597860$

To link to this article: https://doi.org/10.1080/10255842.2019.1597860

\section{Published online: 08 Apr 2019.}

Submit your article to this journal $\pi$

山 Article views: 6

View Crossmark data ¿ 


\title{
Bicuspid aortic valve aortopathies: An hemodynamics characterization in dilated aortas
}

\author{
Diana Oliveira $^{\mathrm{a}}$ (D), Sílvia Aguiar Rosa ${ }^{\mathrm{b}}$, Jorge Tiago ${ }^{\mathrm{a}}$, Rui Cruz Ferreira ${ }^{\mathrm{b}}$, Ana Figueiredo Agapito ${ }^{\mathrm{b}}$ and \\ Adélia Sequeira $^{a}$

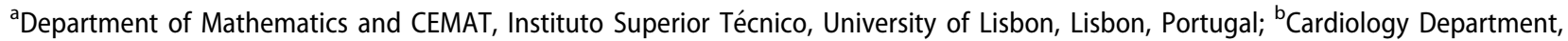 \\ Hospital de Santa Marta (CHLC), Lisboa, Portugal
}

\begin{abstract}
Bicuspid aortic valve (BAV) aortopathy remains of difficult clinical management due to its heterogeneity and further assessment of related aortic hemodynamics is necessary. The aim of this study was to assess systolic hemodynamic indexes and wall stresses in patients with diverse BAV phenotypes and dilated ascending aortas. The aortic geometry was reconstructed from patient-specific images while the aortic valve was generated based on patient-specific measurements. Physiologic material properties and boundary conditions were applied and fully coupled fluid-structure interaction (FSI) analysis were conducted. Our dilated aortic models were characterized by the presence of abnormal hemodynamics with elevated degrees of flow skewness and eccentricity, regardless of BAV morphotype. Retrograde flow was also present. Both features, predicted by flow angle and flow reversal ratios, were consistently higher than those reported for non-dilated aortas. Right-handed helical flow was present, as well as elevated wall shear stress (WSS) on the outer ascending aortic wall. Our results suggest that the abnormal flow associated with BAV may play a role in aortic enlargement and progress it further on already dilated aortas.
\end{abstract}

\section{ARTICLE HISTORY}

Received 31 May 2018

Accepted 18 March 2019

\section{KEYWORDS}

aortic dilation; aorthopathy bicuspid aortic valve; fluidstructure interaction

\section{Introduction}

Bicuspid aortic valve (BAV) is the most common form of congenital heart disease, affecting $0.5 \%$ to $1.4 \%$ of the population (Guntheroth 2008). It has high phenotypic heterogeneity (Kang et al. 2013), associated with valvular pathologies (stenosis, regurgitation), and with the development of ascending aortic dilation (Corte et al. 2006, 2007). The reported prevalence of dilation in these patients ranges from about 30 to $80 \%$ in the adult BAV population (Corte et al. 2007). Pathogenesis of aortic dilation in these patients is still controversial: the genetic basis supports the presence of a congenital defect in the aortic structure, which is backed by histopathological studies showing degeneration of the media layer of the ascending aortic wall (Grewa et al. 2014). The hemodynamic theory argues that the abnormal BAV dynamics causes perturbations on blood flow patterns and hemodynamic stress on the aortic wall, leading to aortic dilation (Mahadevia et al. 2014). According to current knowledge, the high heterogeneous expression of BAV aortopathy suggests that both genetic and hemodynamic factors coexist - a genetic predisposition in these patients may weaken the aortic wall, conferring it with susceptibility for dilation, and the presence of altered hemodynamic might act as a triggering and maintaining factor of that dilation (Padang et al. 2013).

According to this, research studies have tried to underline mechanisms associated with dilation onset and progression in the presence of a BAV. Computational works have provided further insight on the hemodynamic aspects of ascending aortic blood flow in this disease: flow eccentricity, skewness, helical patterns and abnormally elevated wall shear stress (WSS) have been reported by computational (Cao et al. 2017; Bonomi et al. 2015; Kimura et al. 2017) and in vivo studies (Mahadevia et al. 2014; Rodriguez-Palomares et al. 2018). Nonetheless, further hemodynamics characterization of blood patterns in the ascending aorta in cases of dilation is necessary. $4 \mathrm{D}$ flow MRI studies are extensively used to assess aortic hemodynamics in BAV patients (Mahadevia et al. 2014; Rodriguez-Palomares et al. 2018; Hope et al. 2010). However, this imaging modality is unable to evaluate accurately and noninvasively relevant 

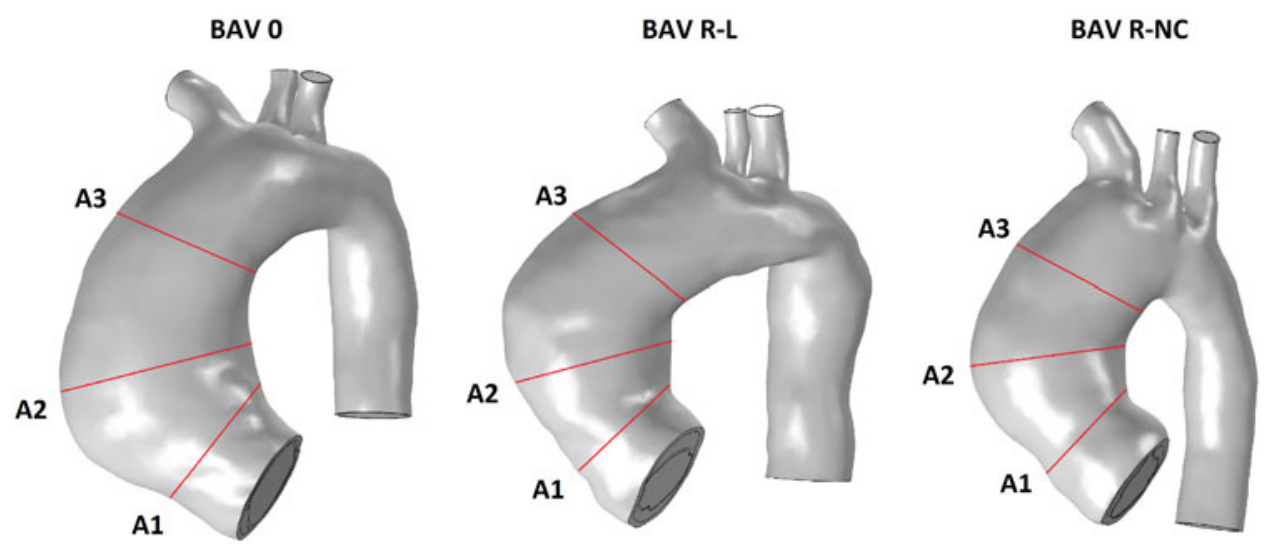

Figure 1. Computational domains for all BAV models.

hemodynamic predictors such as WSS, due to low temporal and spatial resolutions (Markl et al. 2011). Thus, computational modelling becomes advantageous in this matter, providing with several hemodynamic indexes that can further describe the abnormal aortic hemodynamics present in BAV patients.

The purpose of this study is to assess ascending aortic hemodynamics in patients with dilated aortas and different BAV phenotypes. We propose a threedimensional morphologic modelling of bicuspid aortopathies that employs patient-specific aortic geometries and measurements for the geometric reconstruction of the valve leaflets. Then, we characterize aortic hemodynamics by computing indices analysing jet asymmetry, flow reversal, helicity and wall shear stress distributions.

\section{Methods}

\subsection{Data acquisition and model generation}

Three patient-specific non-calcified and non-regurgitant BAV cases were considered: two cusps without a raphe (BAV 0), right-left fusion (BAV R-L) and rightnon-coronary fusion (BAV R-NC), characterized by aortic dilation (aortic diameter $>40 \mathrm{~mm}$ ). Patients underwent computed tomography angiography of the thoracic aorta, scanned on a 64-slice multislice (slice thickness $=1.25 \mathrm{~mm}$ ) computed tomography scanner (LightSpeed VCT XT, GE Healthcare, Milwaukee, USA). The contrast-enhanced scan was obtained using VisipaqueTM (iodixanol) injected using automated pump injectors through a peripheral vein followed by saline bolus chase, in accordance with patient's weight. Three-dimensional surface models of the aortic root (including aortic sinuses), ascending aorta and aortic arch were then created from these images through manual segmentation (3D-Slicer v.4.8.0).
An open-source tool (LifeVFSIMeshGeneration) was used to generate the aortic wall and a thickness proportional to the radius of the lumen was employed (Faggiano et al. 2013a). This tool turns the dimension of local triangular mesh elements proportional to the local lumen radius. This is computed by coupling information about the local distance between the interface surface and the vessel centerlines and the local maximum inscribed sphere radius: this process yields adequate mesh refinement. The mathematical formula employed for radius dependent meshing is

$$
h=\alpha \cdot r^{\beta},
$$

where $h$ is the aortic wall thickness, $r$ is the lumen local radius and $\alpha$ and $\beta$ are fitting parameters. Literature values of wall thickness and lumen diameter were used to estimate $\alpha$ and $\beta$. A two-system equation with wall thicknesses of $1.63 \mathrm{~mm}$ and $0.22 \mathrm{~mm}$ corresponding to lumen diameters of $29 \mathrm{~mm}$ and $3 \mathrm{~mm}$, respectively, was created using the equation above, and fitting parameters were obtained (Reymond et al. 2012; Avolio 1980).

The surfaces at the valve annulus plane and the outlets were cut, yielding the final aortic volumes. Cross-sections at the sinotubular junction (A1), tubular ascending aorta (A2) and beginning of the aortic arch (A3) were defined by creating planes perpendicular to the lumen (Fig. 1).

Previous studies showed that the open systolic configuration of the BAV leaflets is sufficient to recreate the abnormal aortic hemodynamics associated (Bonomi et al. 2015). Since the goal of this work was to study BAV related ascending hemodynamics at systole, BAV leaflets were reconstructed in a fixed peak systolic configuration. All patients underwent a comprehensive Doppler echocardiographic study (Vivid 9; General Electric) and an anatomical assessment of BAV was performed using images from parasternal 
Table 1. Patient-specific measurements retrieved from echocardiographic images.

\begin{tabular}{lcccc}
\hline & \multicolumn{4}{c}{ Dimensions } \\
\cline { 2 - 5 } BAV type & $\begin{array}{c}\text { orifice } \\
\text { area [mm] }\end{array}$ & $\begin{array}{c}\text { ring diameter } \\
{[\mathrm{mm}]}\end{array}$ & $\begin{array}{c}\text { raphe } \\
\text { length [mm] }\end{array}$ & $\begin{array}{c}\text { leaflet } \\
\text { height [mm] }\end{array}$ \\
\hline BAV 0 & 160 & 26.75 & - & 12.37 \\
BAV R-L & 120 & 25.45 & 11.79 & 13 \\
BAV R-NC & 240 & 26.76 & 13.08 & 11.32 \\
\hline
\end{tabular}

long and short axis views. Valve measurements were then retrieved (Table 1). Characteristics impossible to observe in these images (e.g. shape of the raphe) were retrieved from previous computational works (Cao et al. 2016a, 2017), including the use of uniform thickness of $0.5 \mathrm{~mm}$ for all configurations. Leaflets were created using SOLIDWORKS 2016 (Dassault Systemes, Inc, Velizy-Villacoublay, France) (Fig. 2). BAV configurations were then matched to the respective aortic volumes. Their position was adjusted through careful observation of angiographic and echocardiographic images.

\subsection{Fluid-structure interaction framework and grid settings}

The wall deformation of the aorta under the action of the pulsatile blood flow has been pointed as crucial in computational models, due to the compliant properties of this structure (Azadani et al. 2012). In fact, computational results obtained by FSI and computational fluid dynamics analysis may differ, as shown in (Mendez et al. 2018), where distinct stress distributions and mean pressure values were emphasized. Therefore, it is important to have into account the deformation of the ascending aortic wall when modelling its hemodynamics.

A FSI framework was then adopted to model the interaction between blood and the aortic wall. FSI models using the Arbitrary Lagrangian-Eulerian (ALE) formulation (Fernandez et al. 2009) were implemented in COMSOL Multiphysics 5.0.

Blood was approximated as an incompressible, homogeneous and Newtonian fluid (Parker and Thiriet 2009), modelled with the Navier-Stokes equations for mass conservation (eq. 2) and linear momentum (eq. 3).

$$
\begin{gathered}
\nabla \cdot \mathbf{u}=0, \\
\rho\left\{\frac{d \mathbf{u}}{d t}+(\mathbf{u} \nabla) \mathbf{u}\right\}+\nabla P-(\mu \mathbf{D}(\mathbf{u}))=f,
\end{gathered}
$$

where $\mathbf{u}$ is the velocity vector, $\rho$ is the blood density, $\mathrm{P}$ is the pressure, $\mu$ is the dynamic blood viscosity, $f$ accounts for the action of external forces and $\mathbf{D}(\mathbf{u})$ is the strain rate.

Following the work of other authors ((Cao et al. 2016a; Atkins et al. 2014; Lantz et al. 2011)), the aortic wall was modelled as an isotropic, linear and elastic material, characterized by the linear elastic equation (eq. 4),

$$
\rho^{s} \frac{d \eta^{2}}{d t^{2}}-\nabla \cdot(\sigma)=0
$$

where $\rho^{s}$ is the constant density of the wall, $\eta$ is the wall displacement vector and $\sigma$ is the Cauchy stress vector. Two coupling conditions were also considered at the blood-wall interface, ensuring continuity of velocities and traction forces.

The aortic model was meshed with tetrahedral elements. To improve numerical accuracy while minimizing the computational cost, a coarser grid was used for the structure domain and a finer grid was created at the fluid domain. A structured hexahedral mesh was used for boundary layers in the fluid near the FSI interface. A mesh sensitivity analysis was performed. This consisted of measuring the relative errors (computed using the $H^{1}(\Omega)$ semi-norm and the $L^{2}(\Omega)$ norm, where $\Omega$ represents the computational domain) between approximate velocity solutions obtained under increasing mesh density, and the solution obtained with the finest mesh. Mesh refinement was achieved by progressively decreasing element size. A suitable window of values appeared after one million degrees of freedom (DOF) for the fluid domain $\left(L^{2}(\Omega)<3 \% ; H^{1}(\Omega)\right.$ semi-norm between $10 \%$ and $15 \%)$. The final computational grid yielded one million DOF for the fluid domain and an average spatial resolution of $0.0009 \mathrm{~m}$ and $0.0013 \mathrm{~m}$ for the fluid and solid domains, respectively. No remeshing methods were employed, but hyperelastic smoothing of the deformed mesh was applied.

A finite element discretization based on stabilized $\mathrm{P} 1+\mathrm{P} 1$ (Bazilevs et al. 2007) and P2 elements for the fluid and structure domains, respectively, was employed. A fully-coupled (nonlithic) approach was used to solve fluid and solid equations. Each non-linear iteration was tackled with the undamped NewtonRaphson method, with a Jacobian update at each time step and a relative tolerance of 0.01 . Linear iterations were solved with the direct solver PARDISO (www. pardiso-project.org), used for solving large sparse equations. As to LU factorization, the matrix preordering algorithm Nested Dissection Multithreaded was used (Petra et al. 2014). Time discretization was handled with a BDF solver (Hindmarsh et al. 2005) using an adaptative order (up to two) and an adaptive 

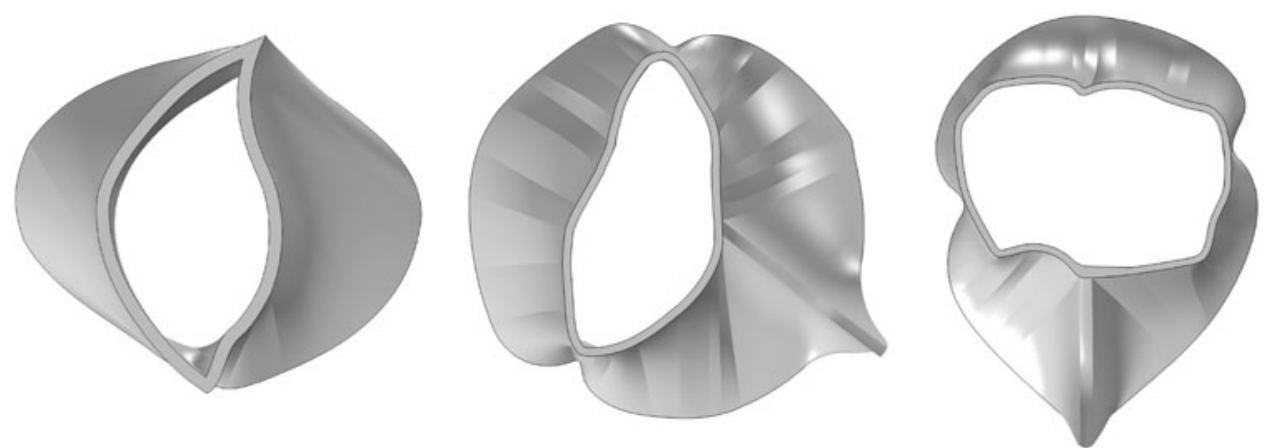

Figure 2. Valve leaflets for BAV 0 (left), BAV R-L (center) and BAV R-NC (right) configurations.

time step up to $4.5 \mathrm{e}-3$ s. All simulations were run over three cardiac cycles to achieve temporal convergence. A workstation with a processor Dual CPU Xeon E5-2630 v3 @2.4GHz and $128 \mathrm{~GB}$ RAM memory was used.

\subsection{Material properties and boundary conditions}

Blood was assumed to have a density of $1050 \mathrm{~kg} / \mathrm{m}^{3}$ and a viscosity of $0.004 \mathrm{~Pa} \mathrm{~s}$. Furthermore, the aortic wall was modelled with a density of $1120 \mathrm{~kg} / \mathrm{m}^{3}$, a Poisson's ratio of 0.45 and a Young's modulus of $2 \mathrm{MPa}$. Moreover, BAV leaflets were modelled as rigid bodies, with prescribed null displacement and rotation.

Patient-specific peak transvalvular flow velocity was obtained by continuous Doppler echocardiography in 5 chambers apical view. Velocity curves were then extrapolated from this data and re-scaled to match the dimensions of each inlet (Fig. 3). Laminar flow was obtained using an artificial entrance extension of six diameters normal to the vessel cross-section.

At the outlets, linear absorbing pressure conditions were applied (Janela et al. 2010), averting spurious backflow from the outlets in a truncated geometrical domain. In addition, the aortic wall was constrained in the longitudinal and circumferential directions at the inlet and outlet sections. A no-slip condition was imposed between blood and wall and no material damping was applied for the latter.

\subsection{Hemodynamic characterization}

Global aortic hemodynamics was assessed at peak systole and during the deceleration phase regarding velocity streamlines and peak systole only for the remaining flow characterizations. Velocity vectors and vorticity fields were captured at sections A2 and A3. Aortic flow helicity was quantified using the positive helix fraction (PHF) index (Cao et al. 2017; Bonomi et al. 2015),

$$
P H F=\frac{H_{p o s}}{H_{\text {pos }}+H_{\text {neg }}},
$$

where $H_{\text {pos }}$ and $H_{\text {neg }}$ are the positive and negative parts, respectively, of the helicity flux over a section,

$$
H=\int_{S} \nabla \times((\mathbf{u} \cdot \mathbf{t}) \mathbf{t}) \cdot \mathbf{n} d S
$$

where $\mathbf{t}$ and $\mathbf{n}$ are the unit vectors tangent and normal to the section $S$, respectively. PHF yields the ratio between right-handed helical flow and the total rotating flow (PHF $=0$ : purely left-handed helical flow; $\mathrm{PHF}=1$ : purely right-handed helical flow).

Flow skewness and eccentricity were evaluated with the flow angle $\theta$ (Cao et al. 2017):

$$
\cos (\theta)=\frac{(\mathbf{u} \cdot \mathbf{n})}{\|\mathbf{u}\|}
$$

To quantify retrograde flows in the ascending aorta, the flow reversal ratio (FRR) index was used (Bonomi et al. 2015),

$$
F R R=\frac{\left|Q_{\text {neg }}\right|}{\left|Q_{\text {pos }}\right|} \%,
$$

where $Q_{n e g}$ and $Q_{p o s}$ represent the backward and forward flow rates on the section of interest, respectively (when FRR equals 0, no retrograde flow is present).

The WSS magnitude was also computed. In Newtonian fluids, the vectorial WSS is defined as:

$$
\mathbf{W S S}=\tau_{n}-\left(\tau_{n} \cdot \mathbf{n}\right) \mathbf{n},
$$

where $\tau_{n}$ is the viscous stress vector (Reymond et al. 2013), given by

$$
\tau_{n}=\mu\left((\nabla \mathbf{u})^{T}+\nabla \mathbf{u}\right) \cdot \mathbf{n},
$$

Global WSS magnitude in the ascending aorta was assessed at peak systole and its circumferential distribution was also quantified at the $\mathrm{A} 2$ and $\mathrm{A} 3$ cross-sections. 


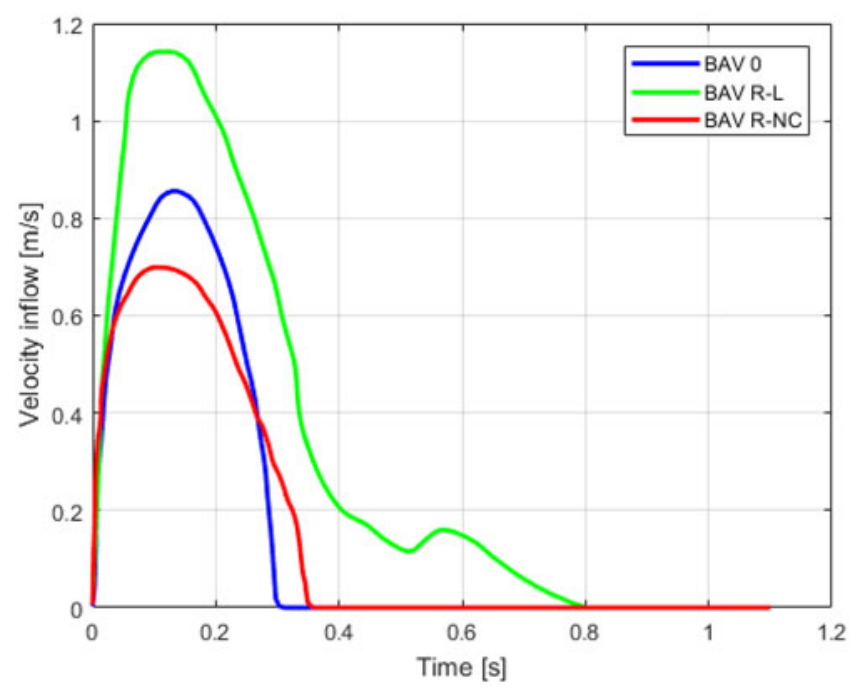

Figure 3. Flow velocity waveforms imposed at the inlet for each BAV patient.

\section{Results}

\subsection{General hemodynamic assessment}

Averaged results for the flow angle, PHF and FRR indexes in our dilated models are presented in Table 2, as well as similar results obtained by previous computational and in vivo studies, in non-dilated and dilated aortas with a BAV.

\subsection{Flow asymmetry}

There is an asymmetric distribution of the velocity field in all models, with peripheral skewing of the systolic jet towards the outer ascending aortic wall (Fig. 4). Flow helices are also present at peak systole, widespreading throughout the deceleration phase.

Fig. 5 depicts elevated degrees of flow skewness and eccentricity in the proximal aorta (A1) for all cases, with $\theta$ varying between $18.2^{\circ}$ and $23.7^{\circ}$. A similar trend is observed at the $\mathrm{A} 2$ and $\mathrm{A} 3$ sections. In A2, however, while skewness decreases for BAV 0 and BAV R-NC $\left(\theta<17.4^{\circ}\right)$, it rises for BAV R-L $(\theta=$ $\left.38.5^{\circ}\right)$. Throughout the ascending aorta, though, the averaged flow angle increases (Table 2).

\subsection{Flow vorticity and helicity}

There is a coexistence of right- and left- helical flow patterns with no prevailing direction in section A1 for all BAV models $(0.48<\mathrm{PHF}<0.51$, Table 2 and Fig. 6). As flow develops along the ascending aorta, prevalent right-handed helices appear in the middle section $(0.58<$ PHF $<0.68)$, associated with intense vortices in all models (maximum values $>250 / \mathrm{s}$, Fig. 7). BAV R-NC presents more marked helical flow
$(\mathrm{PHF}=0.68)$ in comparison with the other two BAV morphotypes (PHF < 0.64), as well as the highest section-averaged vorticity (295.6/s). Right-handed helices spread through distal aorta (A3) $(0.57<$ PHF $<0.65)$.

\subsection{Retrograde flow}

The FRR index quantified in Table 2 and Fig. 8 shows the presence of peak systolic retrograde flow in the ascending aorta for all models: maximums of $18.4 \%$ for BAV 0 and BAV R-L at A1 and $19.3 \%$ for BAV $\mathrm{R}-\mathrm{NC}$ at $\mathrm{A} 2$ are displayed. These express intense recirculation zones throughout the ascending aorta. Such features are diminished but still present at the distal region (A3) $(6.2 \%<F R R<7.4 \%)$.

\subsection{WSS characterization}

High velocity regions correspond to increased WSS magnitudes at the interface between lumen and wall. Due to the BAV asymmetric systolic jet, sites of elevated WSS are observed in the outer wall curvature of the ascending aorta in all models (Fig. 9). Fig. 10 zooms sections A2 and A3 for a better assessment of these characteristics. Positive magnitude values are observed for all models, denoting a streamwise WSS direction. BAVs expose the mid-ascending aortic wall to highly asymmetric WSS, with elevated values observed in the $\mathrm{R}$ and R-A segments (maximum values $>60 \mathrm{dyn} / \mathrm{cm}^{2}$, Fig. 10(a)) and low ones on P, LP, L, L-A and A segments. Distinct features are observed in the A3 section: while asymmetric WSS is still present for the BAV 0 model in a similar fashion, the BAV R-L model yields a different pattern of asymmetry, with maximum values located in the $\mathrm{L}-\mathrm{A}, \mathrm{A}$ and 
Table 2. Hemodynamic predictions for the defined aortic cross-sections and literature results.

\begin{tabular}{|c|c|c|c|c|c|c|c|c|c|}
\hline & \multicolumn{3}{|c|}{ Our study } & \multicolumn{3}{|c|}{$\begin{array}{l}\text { Literature: dilated aortas } \\
\text { (Mahadevia et al. 2014) } \\
\text { (Bonomi et al. 2015) } \\
\text { (Rodriguez-Palomares et al. 2018) }\end{array}$} & \multicolumn{3}{|c|}{$\begin{array}{c}\text { Literature: non-dilated aortas (Bonomi } \\
\text { et al. 2015) } \\
\text { (Cao et al. 2017) } \\
\text { (Rodriguez-Palomares et al. 2018) }\end{array}$} \\
\hline & $\mathrm{A} 1$ & $\mathrm{~A} 2$ & A3 & $\mathrm{A} 1$ & $\mathrm{~A} 2$ & A3 & $\mathrm{A} 1$ & A2 & A3 \\
\hline Flow angle [o] & 21.8 & 23.4 & 29.7 & $22.9-27.2$ & $22.8-30.5$ & $15.8-25.4$ & $11.8-21.1$ & $12.8-23.0$ & 19.5 \\
\hline PHF & 0.5 & 0.63 & 0.6 & & 0.87 & & 0.51 & $0.69-0.72$ & \\
\hline FRR [\%] & 16.3 & 14.0 & 7.0 & 14.2 & $10.8-23.6$ & 11.4 & 11.2 & $11.6-12.3$ & 9.3 \\
\hline
\end{tabular}
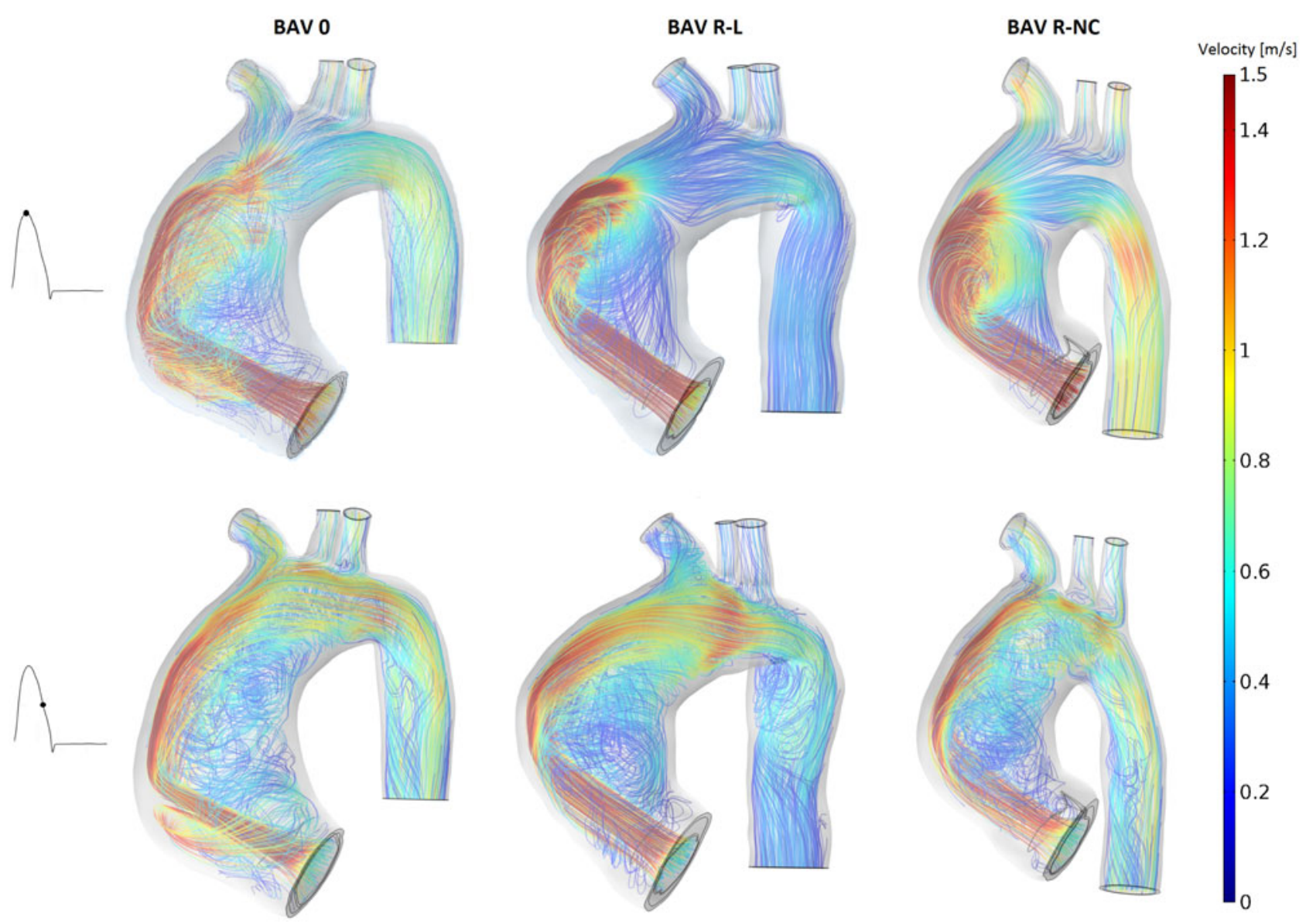

Figure 4. Velocity streamlines in the ascending aorta $[\mathrm{m} / \mathrm{s}]$ at peak systole (first row) and deceleration phase (second row).

R-A segments $\left(<15 \mathrm{dyn} / \mathrm{cm}^{2}\right)$. On the other hand, the BAV R-NC leads to a steadier WSS environment, also associated with the highest section-averaged WSS (A2: $32.7 \mathrm{dyn} / \mathrm{cm}^{2}$; A3: $13 \mathrm{dyn} / \mathrm{cm}^{2}$ ) in comparison with BAV 0 (A2: $\left.29.6 \mathrm{dyn} / \mathrm{cm}^{2} ; \mathrm{A} 3: 11.7 \mathrm{dyn} / \mathrm{cm}^{2}\right)$ and BAV R-L (A2: $19.6 \mathrm{dyn} / \mathrm{cm}^{2} ; \mathrm{A} 3: 7.5 \mathrm{dyn} / \mathrm{cm}^{2}$ ).

\section{Discussion}

\subsection{Design framework and computational model}

Here, we present a study of the hemodynamics associated with BAV dilated aortas, based on patient- specific geometries and valve leaflet measurements. In previous computational analysis of $\mathrm{BAV}$, the aortic model was either built from average dimensions (Cao et al. 2016a, 2017), based on patient-specific magnetic resonance images (MRI) (LaDisa et al. 2010; Faggiano et al. 2013b; Bonomi et al. 2015) or computed tomography angiography images (Pasta et al. 2013; Rinaudo and Pasta 2014; Pasta et al. 2017). BAV leaflets were reconstructed from literature dimensions (Bonomi et al. 2015; Cao et al. 2017, 2016a) or parameterized from medical images (Pasta et al. 2017). Moreover, researchers have split their attention towards the study of hemodynamics in non-dilated BAV aortas 


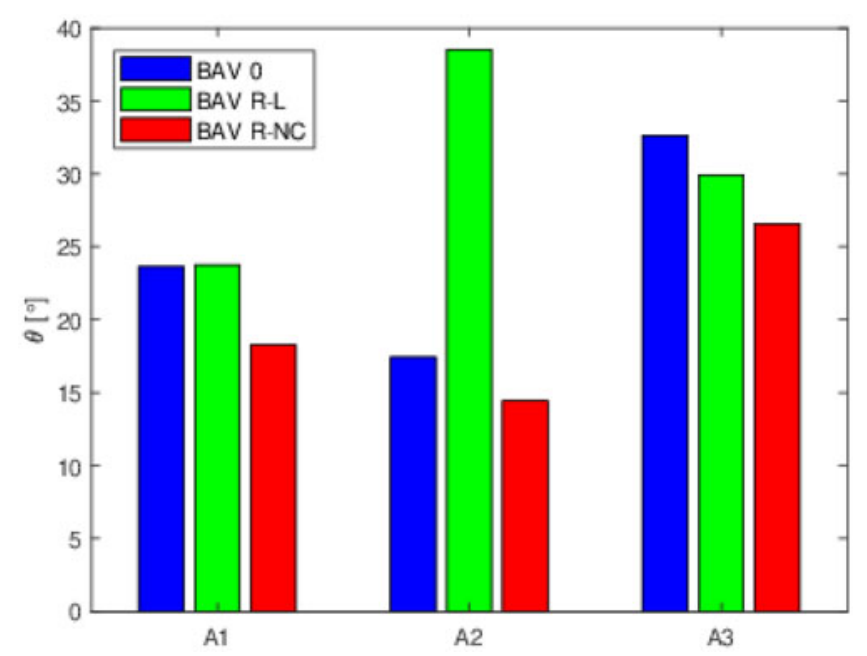

Figure 5. Flow angle $\left[{ }^{\circ}\right]$ predictions through the ascending aorta, at peak systole.

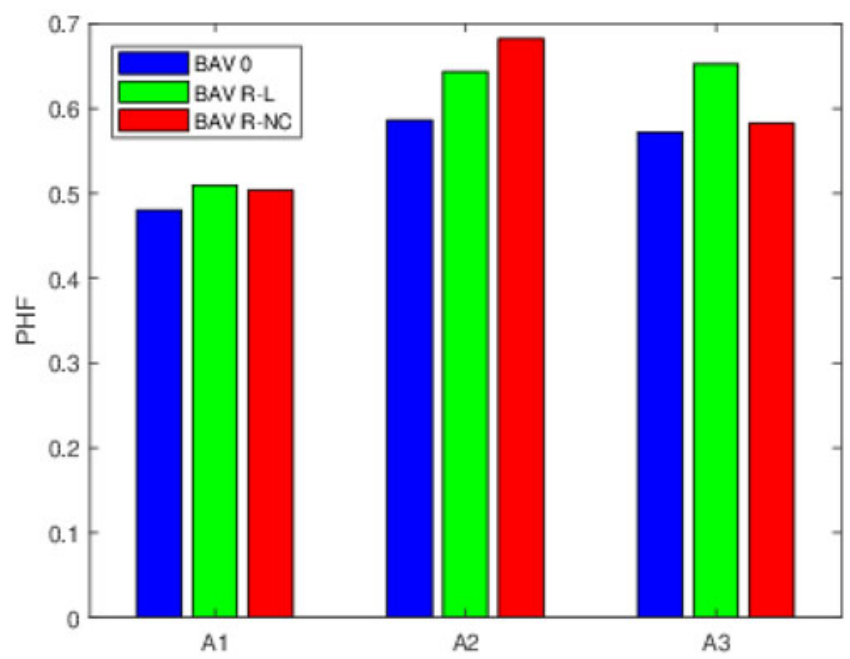

Figure 6. PHF through the ascending aorta, at peak systole.

(Bonomi et al. 2015; Cao et al. 2017; Kimura et al. 2017) and dilated aortas (Bonomi et al. 2015; Pasta et al. 2013; Mendez et al. 2018; Kimura et al. 2017), trying to identify the pathological disturbances that lead to aortic dilation and its progress in these patients.

We modelled the interaction between blood and the aortic wall, but not between blood and the valve leaflets. Instead, we built peak-systolic rigid BAV configurations based on subject-specific measurements and these were mapped into the aortic geometries. In reality, leaflet cyclic motion generates vortical flow in the aortic sinuses during most of systole (Tonimato et al. 2016); therefore, the implementation of nonmoving leaflets most probably eliminated these. However, our study focused on ascending aortic hemodynamics and so that feature was not considered relevant. Moreover, we assumed that the opening/ closure mechanism of the valve should not greatly impact the comparison of systolic fluid-dynamic quantities at the ascending aorta. In addition, the pathophysiology of BAV aortopathy is intrinsically related with the systolic phase. Moreover, none of our patients had aortic regurgitation, present during diastole, which is associated with dilated aortas in BAV patients and is a factor of flow abnormality generation (Keane et al. 2000). Therefore, and since our goal was to study ascending aortic hemodynamics in BAV dilated aortas, we considered the diastolic phase irrelevant and excluded it from this study.

\subsection{BAV induces hemodynamic abnormalities in the ascending aorta}

This study suggests that BAV flow is associated with: (1) the presence of accelerated and skewed systolic blood flow patterns along the outer ascending aortic wall; (2) elevated flow vorticity and helicity in the 


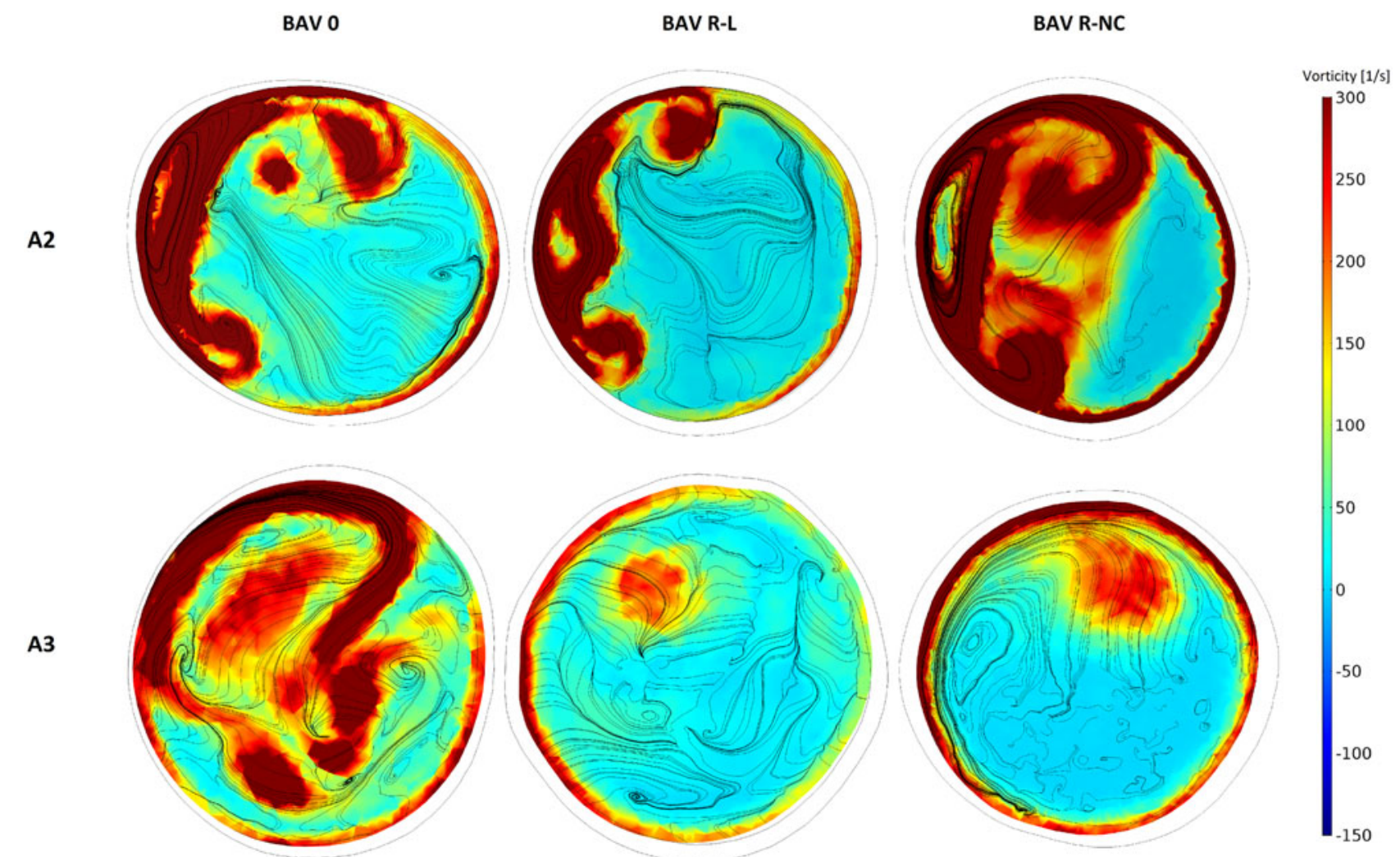

Figure 7. Vorticity [1/s] fields captured in the middle and distal ascending aortic sections.

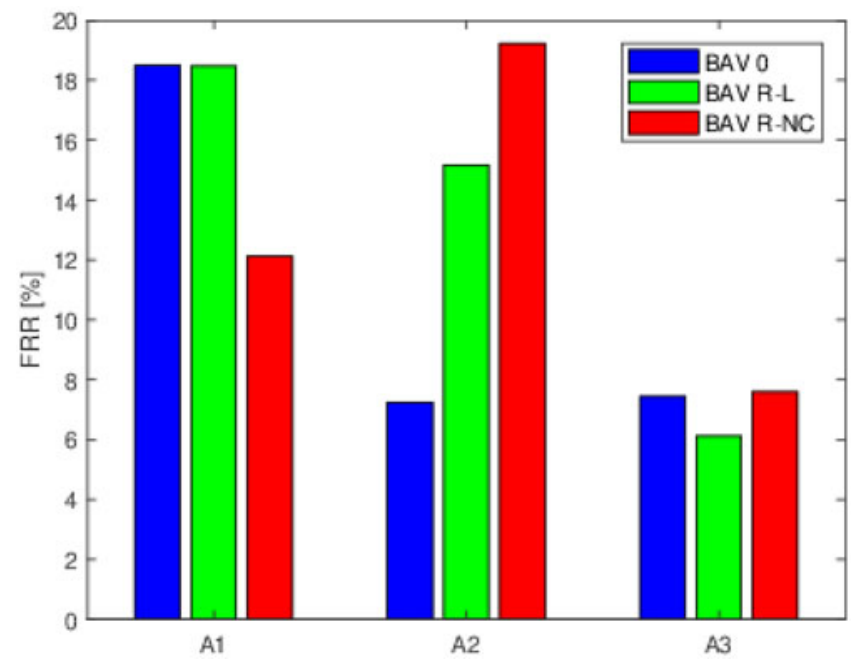

Figure 8. FRR [\%] through the ascending aorta, at peak systole.

ascending aorta; (3) the presence of retrograde flow in the ascending aorta; and (4) elevated WSS magnitude values located at the outer ascending aortic wall. All these findings are consistent with previous computational (Cao et al. 2017, 2016a; Kimura et al. 2017) and clinical (Mahadevia et al. 2014; Barker et al. 2010, 2012; Bissell et al. 2013) works. Expressing the presence of such factors in aortic hemodynamics in patients with a BAV further supports the hypothesis that intrinsic flow abnormalities are implicated in aortic dilation onset and progression, and that dilation is not solely a manifestation of a genetic disorder (Pasta et al. 2013).

\subsection{Does aortic dilation have an impact on BAV flow abnormalities?}

Flow dynamics in dilated BAV aorta is markedly different from a non-dilated one. Flow angles predicted for all BAV models are consistently higher than those 

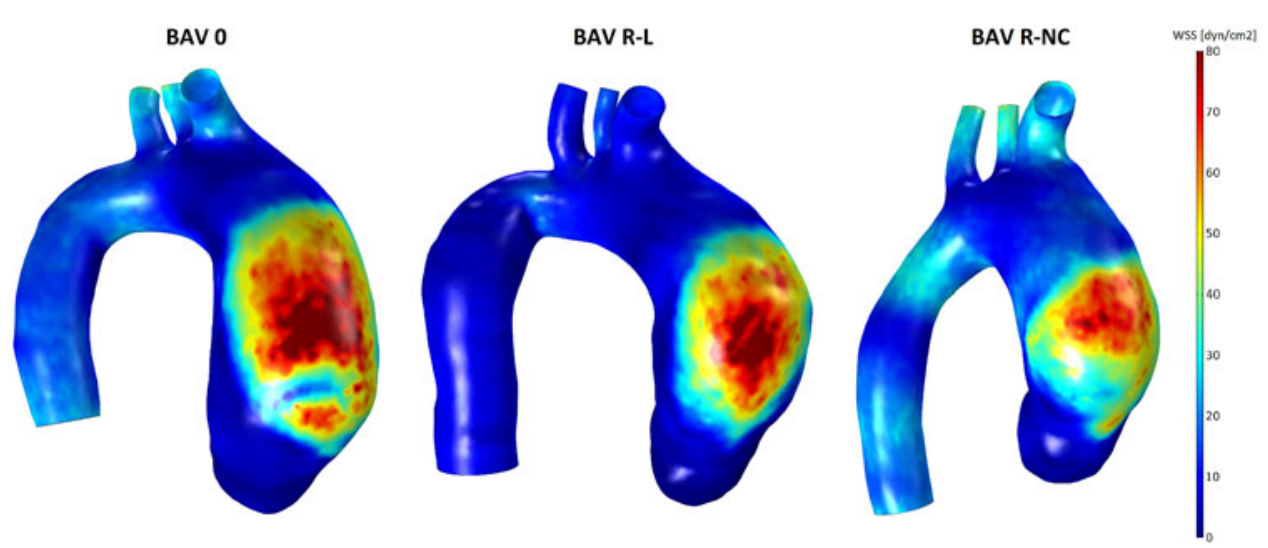

Figure 9. Global WSS magnitude $\left[\mathrm{dyn} / \mathrm{cm}^{2}\right]$ in the ascending aorta, at peak systole.
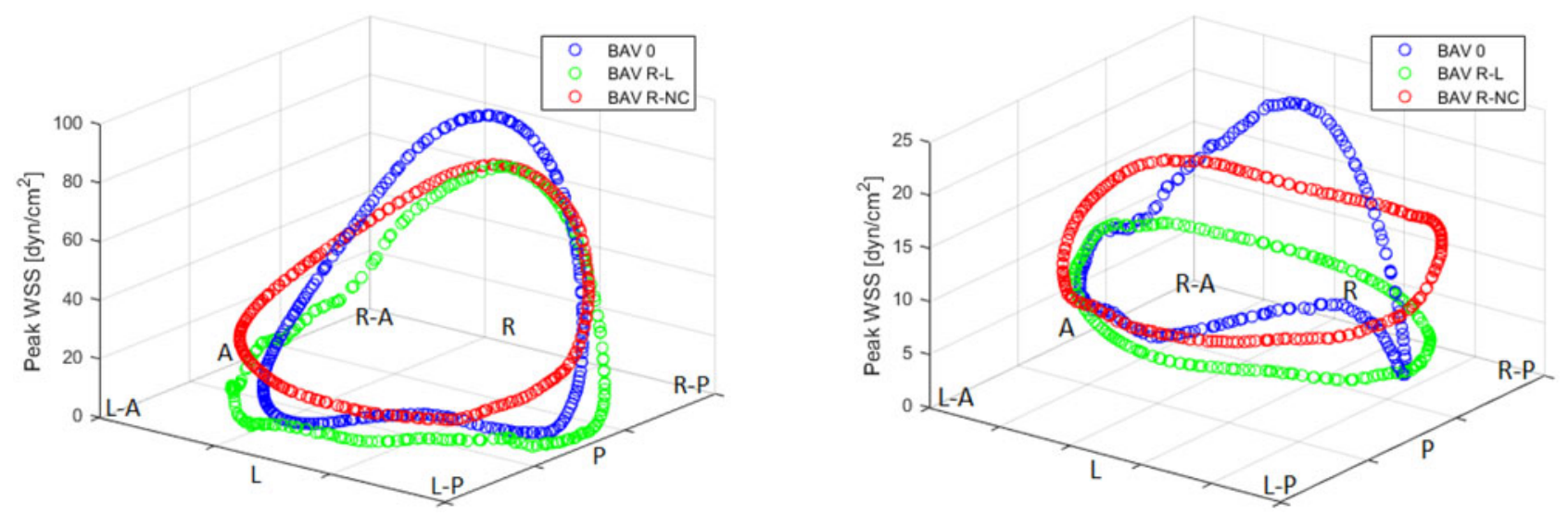

Figure 10. WSS magnitude captured at the two ascending aortic cross-sections, for all BAV models.

reported for non-dilated aortas by other computational studies (Cao et al. 2017) as presented in Table 2. On the other hand, in vivo $4 \mathrm{D}$-flow MRI studies have reported a great variability in flow angle results in dilated (Mahadevia et al. 2014; RodriguezPalomares et al. 2018) and non-dilated (RodriguezPalomares et al. 2018) ascending aortas with a BAV $\left(4^{\circ}\right.$ to $\left.41^{\circ}\right)$. Nonetheless, the flow angle averages reported for dilated aortas also reflect an increase when compared with non-dilated ones and our flow angle predictions do not greatly differ from those obtained in vivo. Our results suggest that ascending aortic dilation may then enhance flow eccentricity.

Alternatively, our study shows a progressive increase of the jet angle through the ascending aorta, which contradicts previous observations ((RodriguezPalomares et al. 2018)), which suggest that flow tends to be more symmetric in the distal ascending aorta. However, we can explain such difference with the fact that we only present results for three BAV aortic models while their study population was much larger.

The right-handed helical flow observed in all BAV dilated models is comparable to the results from previous computational studies either modelling non- dilated (Faggiano et al. 2013b; Bonomi et al. 2015; Cao et al. 2017; Marom et al. 2013) and dilated (Bonomi et al. 2015) aortas. In vivo studies using 4D flow MRI have further depicted helical flow in dilated ascending aortas in the presence of BAV during systole (Mahadevia et al. 2014; Rodriguez-Palomares et al. 2018; Hope et al. 2010)

On the other hand, the PHF index showed a correlation between BAV morphotype and the respective flow rotation type: PHF values had their highest variations reaching $15.9 \%$ between BAV 0 and BAV R$\mathrm{NC}$ at mid-ascending aorta, where BAV R-NC presented with the highest value $(\mathrm{PHF}=0.68)$. Such correlation was not present in studies using non-dilated aortic models (Cao et al. 2017), suggesting that aortic dilation may have an influence in the strength of helical rotations under different BAV phenotypes.

However, the PHF values reported here are lower than the one expressed in a previous study concerning a dilated aortic model: in (Bonomi et al. 2015), highly marked right-handed flow (PHF $>0.84$ ) was observed for a BAV 0 morphotype. This study has actually presented an enhancement in flow helicity in a dilated model in comparison with a non-dilated one 
(see Table 2). Nonetheless, additional data from other computational studies concerning the remaining BAV phenotypes in cases of aortic dilation would be needed to validate our results.

Our dilated aortic models with a BAV also lead to the presence of retrograde flow in the ascending aorta, associated with elevated values of FRR. Other computational (Bonomi et al. 2015; Faggiano et al. 2013b) and in vivo (Rodriguez-Palomares et al. 2018) works also denoted this feature, and an increase in the FRR index on dilated cases versus non-dilated ones. The progressive decrease of this index through the ascending aorta observed in our models is also in correlation with the literature (see Table 2). Nonetheless, such elevated values of FRR are thought to induce an asymmetric increase and directional variations in the WSS in the ascending aorta, contributing to dilation (Rodriguez-Palomares et al. 2018). It is not clear, though, if this parameter is related to the onset of aortic dilation or its progression; however, since retrograde flow is also present in non-dilated aortas (see Table 2), previous studies suggest that such flow can act as a causal agent of dilation, becoming enhanced with greater aortic enlargement and bolstering the dilation process (RodriguezPalomares et al. 2018).

Lastly, flow reversal was dependent on BAV morphology, with the FRR index showing distinct features among BAV types. A previous computational work found a correlation between decreased valve orifice area and increased FRR percentages (Bonomi et al. 2015). This particular reason might explain why several of our FRR percentages (e.g. 19.3\% for BAV R-NC) are more similar to those found in stenotic/ low entrance flow rate models.

Peak WSS magnitudes obtained for our aortic models are in the same range as those reported by other computational studies employing dilated aortas (Pasta et al. 2017) and lower than those obtained with non-dilated models (Cao et al. 2017, 2016a). Similar findings are present in computational (Bonomi et al. 2015) and in vivo studies (Mahadevia et al. 2014; Bissell et al. 2013), where higher degrees of ascending aortic dilation were associated with lower WSS values. Nonetheless, previous 4D flow studies using nondilated aortas have suggested that abnormally high WSS may anticipate the onset of aortopathy and contribute to its triggering (Piatti et al. 2017).

Peak WSS distribution and magnitude was also affected by leaflet fusion type, although asymmetric WSS was present at A2 for all models. Different patterns were seen distally, with the BAV R-NC generating a more moderate/even WSS environment. Nonetheless, previous computational (Cao et al. 2017; Pasta et al. 2017) and clinical (Mahadevia et al. 2014; Barker et al. 2010; Bissell et al. 2013) studies also reported asymmetric regional WSS in patients with distinct BAV morphologies.

\subsection{Clinical applications}

In line with previous works, our study shows that flow abnormalities in the ascending aorta with a BAV are enhanced in dilated cases when compared to nondilated ones, as demonstrated by the quantification of flow asymmetry and retrograde flow, for example. Our computational model can therefore help explain the pathophysiologic process concerning BAV aortopathies through the detailed study of ascending aortic hemodynamics in these cases.

Our findings favor the proposals of previous studies of using hemodynamic characterizations, such as flow angle (Mahadevia et al. 2014), flow asymmetry and the presence of vortices (Faggiano et al. 2013b) as predictors of the localization of elevated WSS in the ascending aorta and consequently as possible indicator of the risk of dilation in these patients. The use of our computational model can aid doctors in risk stratification, by estimating patients with a larger risk for dilation onset and progression.

\section{Limitations}

Some limitations were present in this work. First, this was a pilot study to test the framework developed in a small sample that hindered quantitative statistical comparisons. A large cohort of patients would be needed to 1) validate the results hereby presented; 2) further access the potential of this preliminary framework for clinical translation of data. For completeness, the addition of a non-dilated aortic model with a BAV would have been beneficial for comparison purposes.

Second, the computational model suffers from several limitations. The main one concerns the adoption of rigid leaflets, whose related assumptions have been addressed and discussed. Additionally, leaflet tissue properties were discarded. Future work will be employed towards the inclusion of moving leaflets and the subsequent study of flow patterns in the aortic sinuses derived from leaflet cyclic motion.

Moreover, the aortic wall was built using thickness data from literature. Since patient-specific vessel wall thickness is crucial to accurately predict WSS (Neil 
and Kerckhoffs 2010; Pasta et al. 2013), efforts should be made regarding its use. Finally, the aortic wall tissue was assumed isotropic, although it is anisotropic in behaviour (Pasta et al. 2013).

\section{Conclusions}

Our study demonstrated that: 1) abnormal hemodynamics is present in dilated aortas with a BAV, enhanced in comparison with non-dilated ones, and 2) the flow metrics and WSS regional characteristics employed here can provide insight into that abnormal behaviour. Further validation is necessary: clinical translation depends on large scale validation studies, standardization of technical procedures and costeffectiveness evaluation. Despite the limitations noted above, this non-invasive approach can be relevant to study the impact of transvalvular flow in the ascending aortic wall in BAV patients, providing important information in dilated and non-dilated aortas.

\section{Disclosure statement}

No potential conflict of interest was reported by the authors.

\section{Funding}

The partial financial support from the Portuguese FCT Fundação para a Ciência e a Tecnologia through the grant SFRH/BPD/109574/2015, the project UID/Multi/04621/2013 of the CEMAT - Center for Computational and Stochastic Mathematics, Instituto Superior Técnico, University of Lisbon, Portugal and the UT Austin-Portugal Program (Advanced Computing) are deeply acknowledged.

\section{ORCID}

Diana Oliveira (D) http://orcid.org/0000-0002-8151-1333

\section{References}

Atkins SK, Cao K, Rajamannan NM, Sucosky P. 2014. Bicuspid aortic valve hemodynamics induces abnormal medial remodeling in the convexity of porcine ascending aortas. Biomech Model Mechanobiol. 13(6):1209-1225.

Avolio AP. 1980. Physiological simulation of blood flow in the aorta: Multi-branched model of the human arterial system. Med Biol Eng Comput. 18(6):709-718.

Azadani AN, Chitsaz S, Matthews PB, Jaussaud N, Leung J, Tsinman T, Ge L, Tseng EE. 2012. Comparison of mechanical properties of human ascending aorta and aortic sinuses. Ann Thorac Surg. 93(1):87-94.
Barker AJ, Lanning C, Shandas R. 2010. Quantification of hemodynamic wall shear stress in patients with bicuspid aortic valve using phase-contrast MRI. Ann Biomed Eng. 38(3):788-800.

Barker AJ, Markl M, Bürk J, Lorenz R, Bock J, Bauer S, Schulz-Menger J, von Knobelsdorff-Brenkenhoff F. 2012. Bicuspid aortic valve is associated with altered wall shear stress in the ascending aorta. Circ Cardiovasc Imaging. 5(4):457-466.

Bazilevs Y, Calo V, Tezduyar T, Hughes T. 2007. YZ $\beta$ discontinuity capturing for advection-dominated processes with application to arterial drug delivery. Int J Numer Meth Fluids. 54(6-8):593-608.

Bissell MM, Hess AT, Biasiolli L, Glaze SJ, Loudon M, Pitcher A, Davis A, Prendergast B, Markl M, Barker AJ, et al. 2013. Aortic dilation in bicuspid aortic valve disease: flow pattern is a major contributor and differs with valve fusion type. Circ Cardiovasc Imaging. 6(4): 499-507.

Bonomi D, Vergara C, Faggiano E, Stevanella M, Conti C, Redaelli A, Puppini G, Faggian G, Formaggia L, Luciani GB. 2015. Influence of the aortic valve leaflets on the fluid-dynamics in aorta in presence of a normally functioning bicuspid valve. Biomech Model Mechanobiol. 14(6):1349-1361.

Cao K, Sucosky P. 2016. Computational comparison of regional stress and deformation characteristics in tricuspid and bicuspid aortic valve leaflets. Int J Numer Meth Biomed Engng. 33(3):e02798.

Cao K, BukaC M, Sucosky P. 2016. Three-dimensional macro-scale assessment of regional and temporal wall shear stress characteristics on aortic valve leaflets. Comput Methods Biomech Biomed Engin. 19(6): 603-613.

Cao K, Atkins SK, McNally A, Liu J, Sucosky P. 2017. Simulations of morphotype-dependent hemodynamics in non-dilated bicuspid aortic valve aortas. J Biomech. 50: 63-70.

Corte A, Romano G, Tizzano F, Amarelli C, De Santo L, De Feo M, Scardone M, Dialetto G, Covino F, Cotrufo M. 2006. Echocardiographic anatomy of ascending aorta dilatation: Correlations with aortic valve morphology and function. Int J Cardiol. 113(3):320-326.

Corte A, Bancone C, Quarto C, Dialetto G, Covino F, Scardone M, Caianiello G, Cotrufo M. 2007. Predictors of ascending aortic dilatation with bicuspid aortic valve: a wide spectrum of disease expression. Eur J Cardiothorac. 31:397-405.

Faggiano E, Formaggia L, Antiga L. 2013. An open-source tool for patient-specific fluid-structure vessel mesh generation. Fifth International Symposium on Modelling of Physiological Flows; Chia Laguna, Italy.

Faggiano E, Antiga L, Puppini G, Quarteroni A, Luciani G, Vergara C. 2013. Helical flows and asymmetry of blood jet in dilated ascending aorta with normally functioning bicuspid valve. Biomech Model Mechanobiol. 12(4): 801-813.

Fernandez M, Formaggia L, Gerbeau JF, Quarteroni A. 2009. The derivation of the equations for fluids and structure. In: Formaggia L, Quarteroni A, Veneziani A, editors. Cardiovascular Mathematics. Modeling and 
simulation of the circulatory system. Milano: Springer; $\mathrm{p}$. 76-121.

Grewa N, Groot A, Poelmann R, Klautz R, Lindeman J, Goumans M, Palmen M, Mohamed S, Sievers H, Bogers A, DeRuiter M. 2014. Ascending aorta dilation in association with bicuspid aortic valve: A maturation defect of the aortic wall. J Thorac Cardiovasc Surg. 148(4): $1583-1590$.

Guntheroth WG. 2008. A critical review of the American College of Cardiology/American Heart Association practice guidelines on bicuspid aortic valve with dilated ascending aorta. Am J Cardiol. 102(1):107-110.

Hindmarsh AC, Brown PN, Grant KE, Lee SL, Serban R, Shumaker DE, Woodward CS. 2005. Sundials: Suite of nonlinear and differential/algebraic equation solvers. ACM Trans Math Softw. 31(3):363.

Hope MD, Hope TA, Meadows AK, Ordovas KG, Urbania TH, Alley MT, Higgins CB. 2010. Bicuspid aortic valve: four-dimensional MR evaluation of ascending aortic systolic flow patterns. Radiology. 255(1):53-61.

Janela J, Moura A, Sequeira A. 2010. Absorbing boundary conditions for a 3D non-Newtonian fluid-structure interaction model for blood flow in arteries. J Eng Sci. 48(11): 1332-1349.

Kang J, Song H, Yang D, Baek S, Kim D, Song J, Kang D, Lim T, Song J. 2013. Association between bicuspid aortic valve phenotype and patterns of valvular dysfunction and bicuspid aortopathy: Comprehensive evaluation using MDCT and echocardiography. JACC Cardiovasc Imaging. 6(2):150-161.

Keane MG, Wiegers SE, Plappert T, Pochettino A, Bavaria JE, Sutton MG. 2000. Bicuspid aortic valves are associated with aortic dilatation out of proportion to coexistent valvular lesions. Circulation. 102(19 Suppl 3):III35-III39.

Kimura N, Nakamura M, Komiya K, Nishi S, Yamaguchi A, Tanaka O, Misawa Y, Adachi H, Kawahito K. 2017. Patient-specific assessment of hemodynamics by computational fluid dynamics in patients with bicuspid aortopathy. J Thorac Cardiovasc Surg. 153(4):52-62.

LaDisa JF, Jr, Taylor CA, Feinstein JA. 2010. Aortic coarctation: recent developments in experimental and computational methods to assess treatments for this simple condition. Prog Pediatr Cardiol. 30(1):45-49.

Lantz J, Renner J, Karlsson M. 2011. Wall shear stress in a subject specific human aorta influence of fluid-structure interaction. Int J Appl Mechanics. 3(4):759-778.

Mahadevia R, Barker AJ, Schnell S, Entezari P, Kansal P, Fedak PW, Malaisrie SC, McCarthy P, Collins J, Carr J, Markl M. 2014. Bicuspid aortic cusp fusion morphology alters aortic three-dimensional outflow patterns, wall shear stress, and expression of aortopathy. Circulation. 129(6):673-682.

Markl M, Kilner P, Ebbers T. 2011. Comprehensive 4D velocity mapping of the heart and great vessels by cardiovascular magnetic resonance. J Cardiovasc Magn Reson. 13(7):1-22.

Marom G, Kim HS, Rosenfeld M, Raanani E, Haj-Ali R, Luca A, Pilato M, Scardulla C. 2013. Fully coupled fluidstructure interaction model of congenital bicuspid aortic valves: effect of asymmetry on hemodynamics. Med Biol Eng Comput. 51(8):839-848.
Mendez V, Di Giuseppe M, Pasta S. 2018. Comparison of hemodynamic and structural indices of ascending thoracic aortic aneurysm as predicted by 2 -way FSI, CFD rigid wall simulation and patient-specific displacementbased FEA. Comput Bio Med. 100:221-229.

Neil ML, Kerckhoffs R. 2010. Current progress in patientspecific modeling. Brief Bioinform. 11(1):111-126.

Padang R, Bannon PG, Jeremy R, Richmond DR, Semsarian C, Vallely M, Wilson M, Yan TD. 2013. The genetic and molecular basis of bicuspid aortic valve associated thoracic aortopathy: a link to phenotype heterogeneity. Ann Cardiothorac Surg. 2(1):83-91.

Parker K, Thiriet M. 2009. Physiology and pathology of the cardiovascular system: a physical perspective. In: Formaggia L, Quarteroni A, Veneziani A, editors. Cardiovascular Mathematics. Modeling and simulation of the circulatory system. Milano: Springer; pp. 1-47.

Pasta S, Rinaudo A, Luca A, Pilato M, Scardulla C, Gleason TG, Vorp DA. 2013. Difference in hemodynamic and wall stress of ascending thoracic aortic aneurysms with bicuspid and tricuspid aortic valve. J Biomech. 46(10): 1729-1738.

Pasta S, Gentile G, Raffa GM, Scardulla F, Bellavia D, Luca A, Pilato M, Scardulla C. 2017. Three-dimensional parametric modeling of bicuspid aortopathy and comparison with computational flow predictions. Artif Organs. 41(9): 92-102.

Petra CG, Schenk O, Lubin M, Gäertner K. 2014. An augmented incomplete factorization approach for computing the schur complement in stochastic optimization. SIAM J Sci Comput. 36(2):C139-C162.

Piatti P, Sturla F, Bissell M, Pirola S, Lombardi M, Nesteruk I, Della Corte A, Redaelli A, Votta E. 2017. 4D flow analysis of BAV-related fluid-dynamic alterations: evidences of wall shear stress alterations in absence of clinically-relevant aortic anatomical remodeling. Front Physiol. 8(441):1-17.

Reymond P, Crosetto P, Deparis S, Quarteroni A, Stergiopulos N. 2013. Physiological simulation of blood flow in the aorta: Comparison of hemodynamic indices as predicted by 3-D FSI, 3-D rigid wall and 1-D models. Med Eng Phys. 35(6):784-791.

Richards KE, Deserranno D, Donal E, Greenberg NL, Thomas JD, Garcia MJ. 2004. Influence of structural geometry on the severity of bicuspid aortic stenosis. Am J Physiol Heart Circ Physiol. 287(3):1410-1416.

Rinaudo A, Pasta S. 2014. Regional variation of wall shear stress in ascending thoracic aortic aneurysms. Proc Inst Mech Eng H. 228(6):627-638.

Rodriguez-Palomares JF, Dux-Santoy L, Guala A, Kale A, Maldonado G, Teixido-Tura G, Galian L, Huguet M, Valente F, Gutierrez L, et al. 2018. Aortic flow patterns and wall shear stress maps by $4 \mathrm{D}$-flow cardiovascular magnetic resonance in the assessment of aortic dilatation in bicuspid aortic valve disease. J Cardiovasc Magn Reson. 20(28):1-15.

Tonimato R, Salmon J, Susin F, Ducci A, Burriesci G. 2016. Physiological vortices in the sinuses of Valsalva: An in vitro approach for bio-prosthetic valve. J Biomech. 43(13):2635-2643. 